GANIT J. Bangladesh Math. Soc. (ISSN 1606-3694) 37 (2017) 175-183

\title{
HYDRODYNAMIC MIXED CONVECTION IN A LID-DRIVEN POROUS SQUARE CAVITY WITH INTERNAL HEAT GENERATING ELLIPTIC BLOCK
}

\author{
Abdul Halim Bhuiyan ${ }^{1^{*}}$, M. Jahirul Haque Munshi ${ }^{2}$ \\ ${ }^{1 *}$ Department of Mathematics, Bangladesh University of Engineering and Technology, \\ Dhaka-1000, Bangladesh, ahalim.du@gmail.com \\ ${ }^{2}$ Department of Mathematics, Hamdard University Bangladesh, Hamdard Nagar, \\ Gazaria, Munshigonj-1510, Bangladesh, jahir.buet.bd@gmail.com
}

Received 29.07.2017 Accepted 10.12.2017

\begin{abstract}
Hydrodynamic mixed convection in a lid-driven porous square cavity with internal heat generating elliptic block is numerically simulated in this paper by employing finite element method. The working fluid is assigned as air with a Prandtl number of 0.71 throughout the simulation. The top lid moves left to right at a constant speed $\left(U_{0}\right)$ with cold temperature and while the bottom lid moves right to left at a constant speed $\left(-U_{0}\right)$ with hot temperature. The left wall is heated while the right wall is linearly heated and heat generating elliptic block is placed at the center of square cavity. The effects of governing parameters in the present study are namely, Rayleigh number Ra, Darcy number $D a$, Grash of number Gr. The effects of heat generation and the porosity of the medium on the streamlines, isotherms, temperature profiles, velocity field and average Nusselt numbers are presented in graphical and tabular forms and discussed. An optimum combination of the governing parameters would result in higher heat transfer. Moreover, it is observed that both the Darcy number and moving lid ordination have significant effects on the flow and thermal fields in the enclosure.
\end{abstract}

Keywords: Mixed convection; Heat generation; Lid-driven porous square cavity; Finite Element Method.

\section{NOMENCLATURE}

\begin{tabular}{|c|c|}
\hline $\begin{array}{ll}G \boldsymbol{r} & \text { Grashof number } \\
\boldsymbol{D a} & \text { Darcy number } \\
\boldsymbol{g} & \text { gravitational acceleration } \\
\boldsymbol{L} & \text { length of the cavity } \\
\boldsymbol{N u} & \text { Average Nusselt number } \\
\boldsymbol{P} & \text { dimensional pressure } \\
\boldsymbol{P r} & \text { Prandtl number } \\
\boldsymbol{p} & \text { pressure } \\
\boldsymbol{R} \boldsymbol{a} & \text { Rayleigh number } \\
\boldsymbol{R} \boldsymbol{e} & \text { Reynolds number } \\
\boldsymbol{T} & \text { dimensional temperature } \\
\boldsymbol{U}, \boldsymbol{V} \text { non-dimensional velocity components } \\
\boldsymbol{u}, \boldsymbol{v} & \text { velocity components } \\
\boldsymbol{X}, \boldsymbol{Y} \text { non-dimensional Cartesian coordinates }\end{array}$ & $\begin{array}{ll}\boldsymbol{x}, \boldsymbol{y} & \text { Cartesian coordinates } \\
q & \text { volumetric rate of heat generation } \\
Q \quad \text { dimensionless heat generation parameter } \\
\text { Greek symbols } \\
\boldsymbol{\alpha} \quad \text { thermal diffusivity } \\
\boldsymbol{\beta} \quad \text { volumetric coefficient of thermal expansion } \\
\boldsymbol{v} \quad \text { kinematic viscosity of the fluid } \\
\boldsymbol{\theta} \quad \text { non-dimensional temperature } \\
\boldsymbol{\rho} \quad \text { density of the fluid } \\
\psi \quad \text { non-dimensional stream function } \\
\text { Subscripts } \\
\boldsymbol{c} \quad \text { cold wall } \\
\boldsymbol{h} \quad \text { hot wall } \\
\boldsymbol{a v e} & \text { average }\end{array}$ \\
\hline
\end{tabular}




\section{Introduction}

A mixed convection problem with lid-driven porous medium fluid flows within an enclosure finds a wide range of applications in various fields of engineering and sciences. In this context, buoyancy-driven phenomena in porous media are actively under investigation. Non-Darcy effects on natural convection in porous media have received a great deal of attention in recent years. This is due to a large number of technical applications, such as, separation processes in chemical industries, filtration, solar collectors, heat exchangers, etc. Researchers have studied the concept from various perspectives. For example, Kaviany [1], Nield and Bejan [2], Vafai [3].Analysis of momentum and energy transfer in a lid-driven cavity filled with a porous medium is investigated numerically by Al-Amiri [4]. Aydan et al. [5] studied numerically the steady, laminar mixed convection heat transfer from an is flux vertical impermeable plate embedded in a fluid-saturated porous medium. Hossain et al. [6] studied natural convection flow in a fluid-saturated porous medium enclosed by non-isothermal walls with heat generation. Basak et al. [7] analysis of mixed convection in a lid-driven porous square cavity with linearly heated side wall(s). It was found the local Nusselt number is 1 at the edges of the bottom wall due to linearly heated side walls. For low Darcy numbers, the local heat transfer rate is almost constant due to symmetric isotherms illustrating natural convection or conduction dominance. Munshi et al. [8] performed a simulation of mixed convection double lid-driven square cavity with inside elliptic heated block. Khanafer et al. [9] performed a simulation of double-diffusive mixed convection in a lid-driven enclosure filled with a fluid-saturated porous medium. Oztop [10] investigated numerically combined convection heat transfer in a porous lid-driven enclosure due to heater with finite length. Rahman et al. [11] numerically studied in Magneto hydrodynamic Mixed Convection around a Heat Conducting Horizontal Circular Cylinder in a Rectangular Lid-driven Cavity with Joule Heating and also same authors investigated [12] numerical study on the conjugate effect of joule heating and magnatohydrodynamics mixed convection in an obstructed lid-driven square cavity. Basak et al. [13] using a numerical simulation investigated natural convection in a square cavity filled with a porous medium: Effects of various thermal boundary conditions. In the light of the above literature review, it appears that no significant work was reported on present study on hydrodynamic mixed convection in a lid driven porous square cavity with internal heat generating elliptic block.

\section{Formulation of the Problem}

The physical model is considered two-dimensional hydrodynamic mixed convection in a lid-driven porous square cavity with internal heat generating elliptic block shown in Fig. 1.

The bottom wall of the porous square cavity is kept at a uniform hot temperature $T_{h}$, whereas the top wall is kept at a constant cold temperature of $T_{c}$. The left wall is kept at hot temperature $T_{h}$ and right wall is linearly heated. The top lid is considered to move from left to right with a uniform speed $U_{0}$ and bottom lid is considered to move from right to left with a uniform speed $-U_{0}$. Here length of the square enclosure is $L$. 


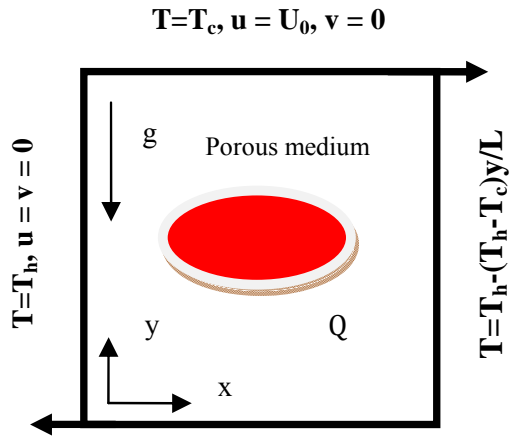

$\mathbf{T}=\mathbf{T}_{\mathrm{h}}, \mathbf{u}=-\mathbf{U}_{\mathbf{0}}, \mathbf{v}=\mathbf{0}$

Fig. 1: The flow configuration and coordinate system.

\section{Governing Equations}

The governing equations for mass, momentum and energy of a steady two-dimensional mixed convection flow in a porous square cavity are as follows:

Continuity Equation

$$
\frac{\partial u}{\partial x}+\frac{\partial v}{\partial y}=0
$$

Momentum Equations

$$
\begin{aligned}
& u \frac{\partial u}{\partial x}+v \frac{\partial u}{\partial y}=-\frac{1}{\rho} \frac{\partial p}{\partial x}+v\left(\frac{\partial^{2} u}{\partial x^{2}}+\frac{\partial^{2} u}{\partial y^{2}}\right)-\frac{v}{\mathrm{~K}} u \\
& u \frac{\partial v}{\partial x}+v \frac{\partial v}{\partial y}=-\frac{1}{\rho} \frac{\partial p}{\partial y}+v\left(\frac{\partial^{2} v}{\partial x^{2}}+\frac{\partial^{2} v}{\partial y^{2}}\right)-\frac{v}{\mathrm{~K}} v+g \beta\left(T-T_{c}\right)
\end{aligned}
$$

Energy Equations

$$
\begin{aligned}
& u \frac{\partial T}{\partial x}+v \frac{\partial T}{\partial y}=\alpha\left(\frac{\partial^{2} T}{\partial x^{2}}+\frac{\partial^{2} T}{\partial y^{2}}\right) \\
& \left(\frac{\partial^{2} T_{S}}{\partial x^{2}}+\frac{\partial^{2} T_{s}}{\partial y^{2}}\right)+q=0
\end{aligned}
$$

where $x, y$ are the distance measured along the horizontal and vertical directions, $u, v$ are the velocity components in the $x$ and $y$ directions, $\rho$ is the density, $p$ is the pressure, $v$ is the kinematic viscosity, $k$ is the permeability of the porous medium, $g$ is the acceleration due to gravity, $\alpha$ is the thermal diffusivity, $\beta$ is the volume expansion coefficient, $T$ is the temperature.

\section{Boundary Conditions}

The boundary conditions for the present problem are specified as follows:

At the top wall: $T=T_{c}, u=U_{0}, v=0$ 
At the bottom wall: $T=T_{h}, u=-U_{0}, v=0$

At right wall: $T=T_{h}-\left(T_{h}-T_{c}\right) \frac{y}{L}, u=0, v=0$

At the left wall: $T=T_{h}, u=0, v=0$

At the elliptic obstacle: $T_{s}=T_{h}, u=0, v=0$

The following dimensionless variables are used to obtain the above equations in non- dimensional form.

$$
\begin{aligned}
& X=\frac{x}{L}, Y=\frac{y}{L}, U=\frac{u L}{U_{0}}, V=\frac{v L}{U_{0}}, \theta=\frac{T-T_{C}}{T_{h}-T_{c}}, \theta_{S}=\frac{T_{S}-T_{C}}{T_{h}-T_{c}} \\
& P=\frac{p L^{2}}{\rho U_{0}^{2}}, \operatorname{Pr}=\frac{v}{\alpha}, D a=\frac{K}{L^{2}}, R e=\frac{U_{0} L}{v}, G r=\frac{g \beta\left(T_{h}-T_{c}\right) L^{3}}{v^{2}}, Q=q L^{2} / k_{f} \Delta T
\end{aligned}
$$

Introducing the above dimensionless variables, the following dimensionless forms of the governing equations are obtained as follows:

Continuity Equation

$$
\frac{\partial U}{\partial X}+\frac{\partial V}{\partial Y}=0
$$

Momentum Equations

$$
\begin{aligned}
& U \frac{\partial U}{\partial X}+V \frac{\partial U}{\partial Y}=-\frac{\partial P}{\partial X}+\frac{1}{R e}\left(\frac{\partial^{2} U}{\partial X^{2}}+\frac{\partial^{2} U}{\partial Y^{2}}\right)-\frac{P_{r}}{D a} U \\
& U \frac{\partial V}{\partial X}+V \frac{\partial V}{\partial Y}=-\frac{\partial P}{\partial Y}+\frac{1}{R e}\left(\frac{\partial^{2} V}{\partial X^{2}}+\frac{\partial^{2} V}{\partial Y^{2}}\right)-\frac{P_{r}}{D a} V+\operatorname{RaPr} \theta
\end{aligned}
$$

Energy Equations

$$
\begin{aligned}
& U \frac{\partial \theta}{\partial X}+V \frac{\partial \theta}{\partial Y}=\frac{1}{R e P r}\left(\frac{\partial^{2} \theta}{\partial X^{2}}+\frac{\partial^{2} \theta}{\partial Y^{2}}\right) \\
& \frac{\partial^{2} \theta_{S}}{\partial X^{2}}+\frac{\partial^{2} \theta_{S}}{\partial Y^{2}}+Q=0
\end{aligned}
$$

\section{The transformed boundary conditions are:}

At the top wall: $\theta=0, U=1, V=0$

At the bottom wall: $\theta=1, U=-1, V=0$

At the left wall: $\theta=1, U=0, V=0$

At the right wall: $\theta=1-Y, U=0, V=0$

At the elliptic obstacle: $U=0, V=0, \theta_{s}=1$

The numerical solutions obtained in terms of the velocity components $(U, V)$ and stream functions $(\psi)$ are defined as follows $U=\frac{\partial \psi}{\partial Y}$ and $V=-\frac{\partial \psi}{\partial X}$. It may be noted that, the positive sign of $(\psi)$ denotes anti-clockwise circulation and the clockwise circulation is represented by the negative sign of $(\psi)$. The no-slip condition is valid at all boundaries as there is no cross-flow, hence $\psi=0$ is used for the boundaries except the moving top wall. To computational of the rate of heat transfer along the hot wall of the enclosure is as follows: 


$$
N u_{\text {local }}=-\left.\frac{\partial \theta}{\partial Y}\right|_{Y=o}
$$

The average Nusselt number of the hot wall is obtained as follows:

$$
N u=\int_{0}^{1} N u_{\text {local }} d X
$$

\section{Grid Refinement Check}

In order to determine the proper grid size for this study, a grid independence test is conducted with five types of mesh for $R e=100, P r=0.71, G r=10^{5}$ and $\mathrm{Da}=1 \mathrm{e}-5$. The extreme value of $\mathrm{Nu}$ is used as a sensitivity measure of the accuracy of the solution and is selected as the monitoring variable. Considering both the accuracy of numerical value and computational time, the present calculations are performed with 18718 nodes and 9850 elements grid system. This is described in Fig. 2(c).

Table 1: Different nodes, elements, average fluid temperature and average Nusselt number for $R e=100$, $\operatorname{Pr}=0.71, G r=10^{5}$ and $D a=1 \mathrm{e}-5$.

\begin{tabular}{|l|l|l|l|l|l|}
\hline Nodes & 26018 & 18718 & 6992 & 2884 & 1850 \\
\hline Elements & 11857 & 8150 & 3409 & 1960 & 1341 \\
\hline$\theta_{\text {ave }}$ & 0.60007 & 0.59818 & 0.57011 & 0.56131 & 0.55461 \\
\hline $\mathrm{Nu}$ & 2.6334 & 2.54602 & 2.26447 & 1.7397 & 1.54208 \\
\hline
\end{tabular}

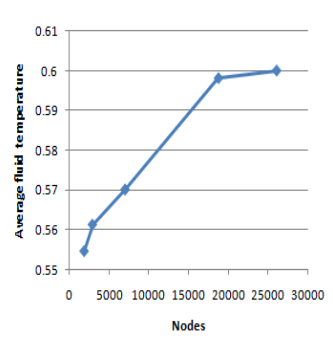

(a)

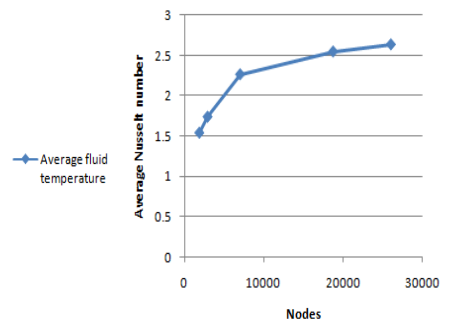

(b)

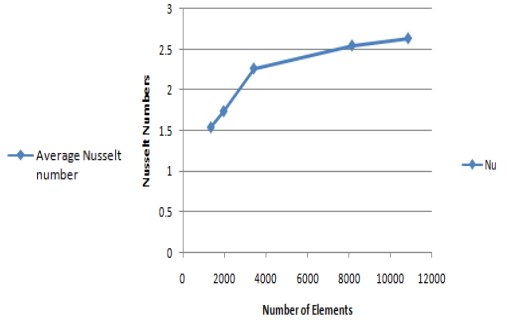

(c)

Fig. 2: Effect of grid refinement test on (a) average fluid temperature $\left(\theta_{\text {ave }}\right)$ verses nodes (b) average Nusselt number verses nodes and (c)average Nusselt number verses Elements while $D a=1 \mathrm{e}-5, G r=10^{5}$, $\operatorname{Re}=100$ and $\operatorname{Pr}=0.71$.

\section{Mesh Generations}

In finite element method, the mesh generation is the technique to subdivide a domain into a set of sub-domains, called finite elements. The discrete locations are defined by the numerical grid, at which the variables are to be calculated. It is basically a discrete representation of the geometric domain on which the problem is to be solved. Mesh generation of elements for the considered cavity is shown in Figure 3. To determine the proper grid size for this study, a grid independence 
test is conduced five types of mesh for $\operatorname{Pr}=0.71, \mathrm{Da}=1 \mathrm{e}-5, \mathrm{Gr}=10^{5}$ and $\operatorname{Re}=100$ which are shown in Table 1.

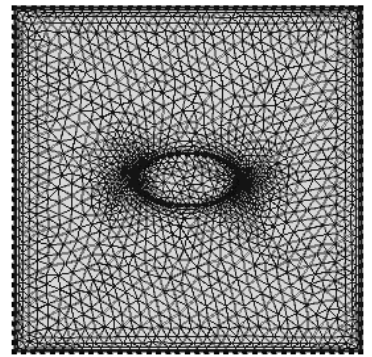

Fig.3: Mess generation in a lid-driven porous square cavity with internal heat generating elliptic block.

\section{Results and Discussions}

A numerical study has been performed to simulate hydrodynamic mixed convection flow in a liddriven porous square cavity with internal heat generation elliptic block. With this aim, results are presented in streamlines, isotherms, temperature, velocity, Local Nusselt number and average Nusselt number in next parts of the work.

Streamlines and isotherms for $D a=1 \mathrm{e}-5$ to $1 \mathrm{e}-2$ are presented in Fig. 4 respectively to understand the effect of Darcy number on the flow field and temperature distribution. When $D a=1 \mathrm{e}-5$ the strength of the buoyancy inside the cavity is significant and more fluid rise from the upper and lower half of the cavity. As $D a$ increases, the strength of the buoyancy increases. Also $D a$ increases to 1e-4 strength of the buoyancy increases and elliptic shape eyes created inside the cavity. Finally, for higher values of $D a$, there is a strong effect of buoyancy force in the flow field and flow strength increasing. Conduction dominant heat transfer is observed from the isotherms in Fig. 4(b) at $D a=1 \mathrm{e}-5$ to $1 \mathrm{e}-2$. With increase in Darcy number, isotherms are symmetrical value about the vertical centre line as the heat generating ellipse block is symmetrical. Also Darcy increase to $1 \mathrm{e}-2$ the isotherms line bending upper and lower wall of the square cavity which means increasing heat transfer through convection. So isotherms lines are developed in these cases.

Streamlines for different values of Grashof number are presented in Fig. 5(a). For lower values of Grashof number two elliptic shape eyesform at the top and bottom portion of the cavity. This circulation cell is formed because of the mixing of the fluid due to the motion of the moving lid and linearly heated side walls. For higher Grashof number more circulation cell is formed inside the cavity and also flow strength increases are shown in Fig. 5(a). Isotherms for different values of Grashof number are presented in Fig. 5(b). For smaller value of Grashof number the isotherms line are smaller changed and parallel to the bottom wall of the cavity. With increasing in Grashof number isotherms are concentrates near the top and bottom wall and isotherms lines are more bending which means increasing heat transfer through convection and moving lid becomes very strong. 
Variations of the vertical velocity component along the bottom wall for different Grashof number with $P r=0.71$ and $D a=1 \mathrm{e}-5$ are shown in Fig. 6(a). It can be seen from the figure that the lower value of Grashof number the value of velocity has smaller changed but for higher Grasof number value of velocity has larger changed. Fig. 6(b) presents the temperature profile along the bottom wall for different Grashof number with $\mathrm{Pr}=0.71$ and $\mathrm{Da}=1 \mathrm{e}-5$. As seen from the Fig. 6(b) temperature value is increased from the increasing of Grashof number. For lower Grashof number value of temperature has smaller significant change as conduction is dominant in this regard.

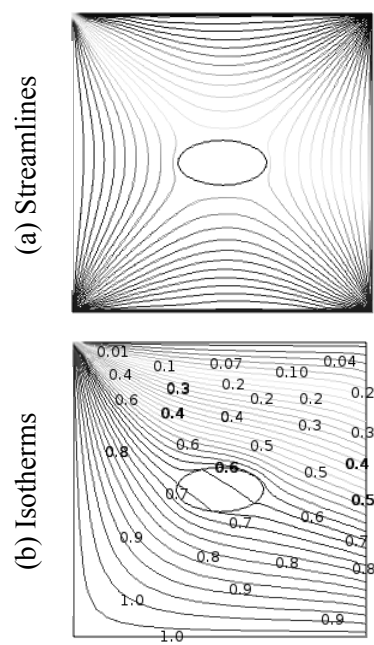

$\mathrm{Da}=1 \mathrm{e}-5$
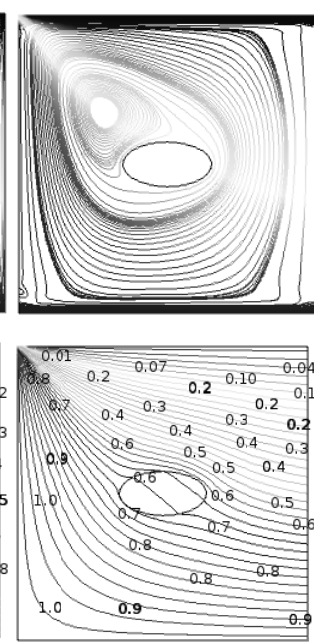

$\mathrm{Da}=1 \mathrm{e}-4$
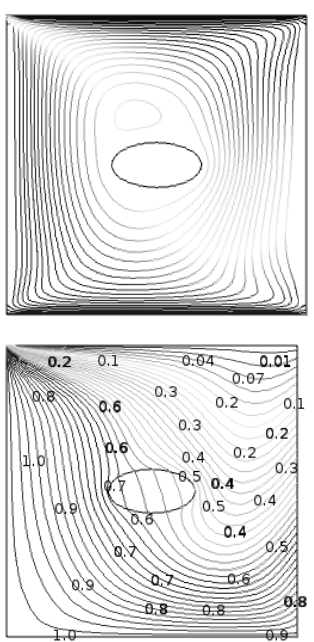

$\mathrm{Da}=1 \mathrm{e}-3$
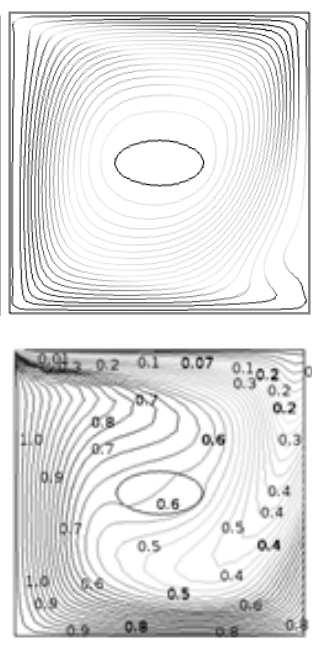

$\mathrm{Da}=1 \mathrm{e}-2$

Fig. 4: (a) Streamlines and (b) Isotherms for different values of Darcy number $D a=1 \mathrm{e}-5$ to $1 \mathrm{e}-2$ when $G r=10^{5}, R e=100, \operatorname{Pr}=0.71$.

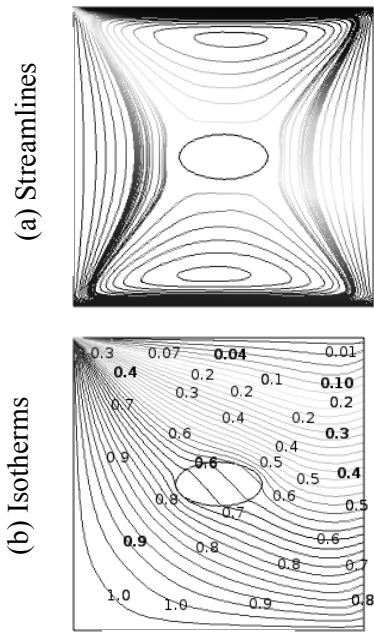

$\mathrm{Gr}=10^{3}$
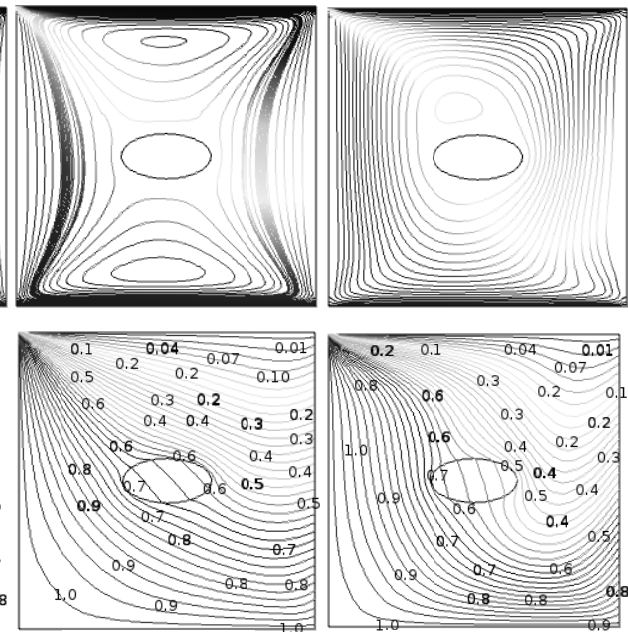

$\mathrm{Gr}=10^{4}$

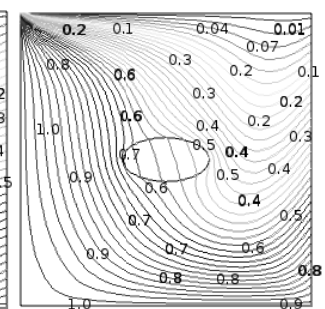

$\mathrm{Gr}=10^{5}$
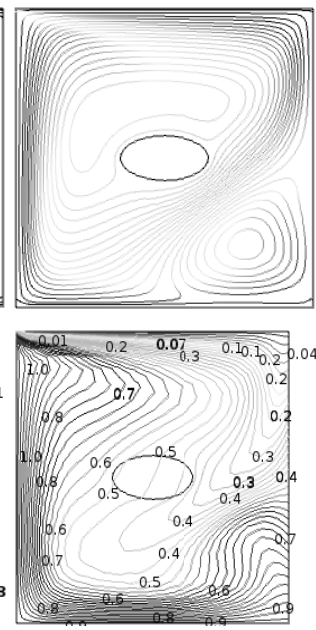

$\mathrm{Gr}=10^{6}$

Fig. 5: (a) Streamlines and (b) Isotherms for different values of Grashof numbers $G r=10^{3}$ to $10^{6}$, when $D a=1 \mathrm{e}-5, R e=100, \operatorname{Pr}=0.71$. 

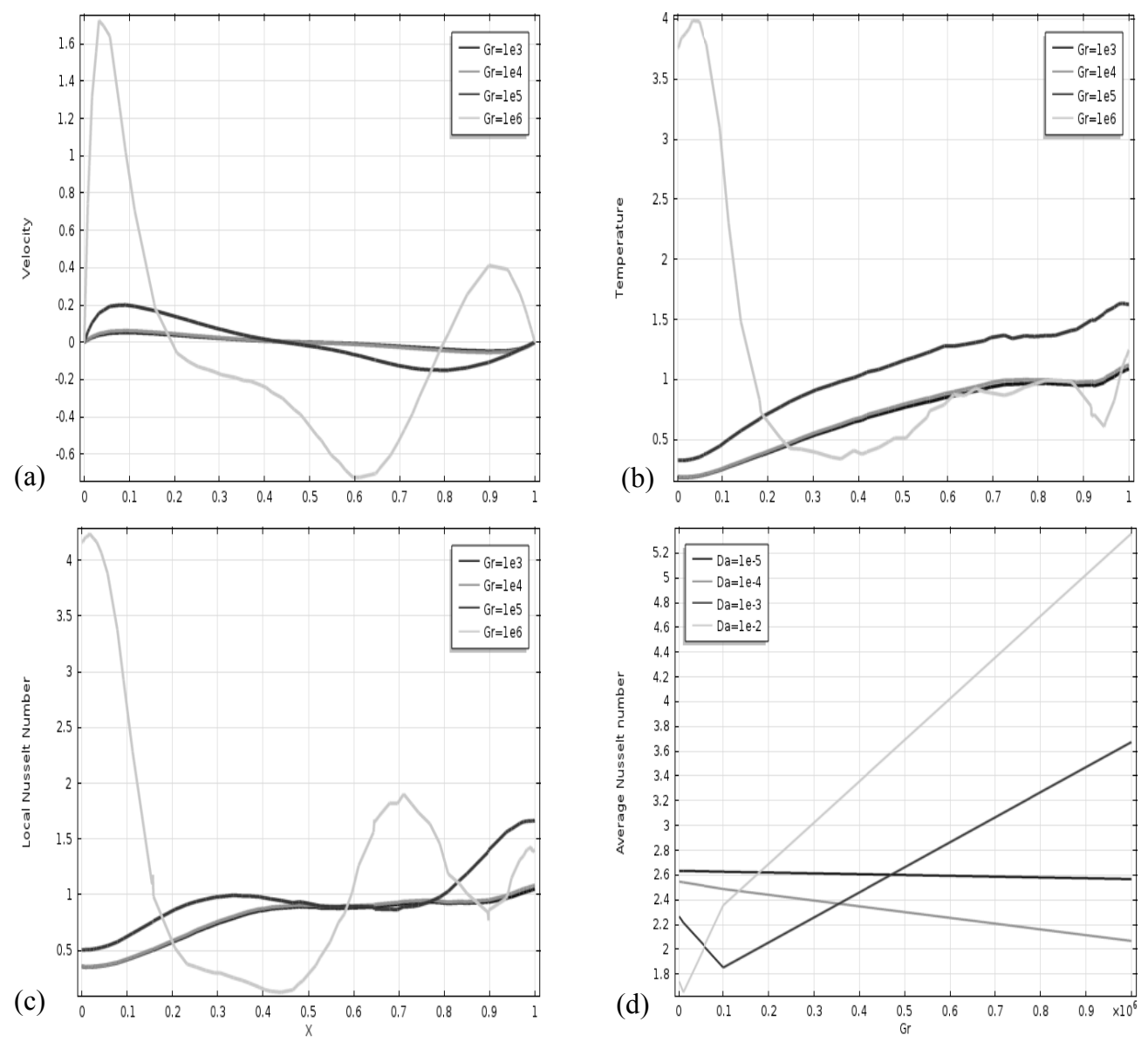

Fig. 6: Variation of (a) Velocity (b) Dimensionless Temperature (c) Local Nusselt number (d) Average Nusselt number versus Darcy number for different Grashof number along the right wall.

Table 2: Average Nusselt number versus Darcy number for different Grashof numbers

\begin{tabular}{|l|l|l|l|l|}
\hline & $D a=1 \mathrm{e}-5$ & $D a=1 \mathrm{e}-4$ & $D a=1 \mathrm{e}-3$ & $D a=1 \mathrm{e}-2$ \\
\hline$G r=1 \mathrm{e} 3$ & 2.6334 & 2.54602 & 2.26447 & 1.7397 \\
\hline$G r=1 \mathrm{e} 4$ & 2.63303 & 2.54118 & 2.21536 & 1.6525 \\
\hline$G r=1 \mathrm{e} 5$ & 2.62654 & 2.48676 & 1.85266 & 2.35577 \\
\hline$G r=1 \mathrm{e} 6$ & 2.56732 & 2.06767 & 3.6735 & 5.35832 \\
\hline
\end{tabular}

The local Nusselt number along the bottom wall for different Grashof number with $\mathrm{Pr}=0.71$ of the cavity are shown in Fig. 6(c). Parabolic shape curves are obtained here. Also it can be seen from the Fig. 6(c) that the local Nusselt number increases with the increasing Grashof number. Plot of the average Nusselt number along the bottom wall as a function of Grashof number at different 
Darcy numbers is show in Fig. 6(d). For high Darcy number, the average Nusselt number increases and the rate of heat transfer is enhanced.

\section{Conclusion}

This paper presents the results of a numerical study hydrodynamic mixed convection in a liddriven porous square cavity with internal hear generating block. The obtained results showed that the heat transfer mechanisms, temperature distribution and the flow characteristics inside the cavity depend strongly both the buoyancy force and the porous medium. For higher values of the Grashof number and the Darcy number, the streamlines and isotherms are distributed strongly in the enclosed domain and the heat is transferred due to convection. Both Darcy and moving lid ordinations have a significant effect on the flow and thermal fields in the temperature.

\section{REFERENCES}

[1] Kaviany, S. (1995): Principles of Heat Transfer in Porous Media, Springer-Verlag, New York.

[2] Nield, D. A. Bejan, A., (1999): Convection in Porous Media, Springer-Verlag, New York.

[3] Vafai, K. (2000): Handbook of Porous Media, Marcel Dekker, New York.

[4] Al-Amiri, A. M. (2000): Analysis of momentum and energy transfer in a lid-driven cavity filled with a porous medium, International Journal of Heat and Mass Transfer, 43: 3513-3527.

[5] Aydin, O., Kaya, A. (2008): Mixed convection of a viscous dissipative fluid about a vertical flat plate embedded in a porous medium: constant heat flux case, J. Porous Media, 11: 207-217.

[6] Anwar Hossain, M., Wilson, M. (2002): Natural Convection Flow in a Fluid-saturated Porous Medium Enclosed by Non-isothermal Walls with Heat Generation, Int. J. Therm. Sci., 41: 447-454.

[7] Basak, T., Roy, S., Singh, S.K., Pop, I. (2010): Analysis of mixed convection in a lid-driven porous square cavity with linearly heated side wall(s), Int. J. Heat and Mass Transfer., 53: 1819-1840.

[8] Jahirul Haque Munshi, M., Alim, M. A. (2016): Effect of Hydromagnetic Mixed Convection Double Lid Driven Square Cavity with Inside Elliptic Heated Block, Journal of Scientific Research, 9(1): 111.

[9] Khanafer, K., Vafai, K. (2002): Double-diffusive mixed convection in a lid-driven enclosure filled with a fluid-saturated porous medium, Numer. Heat Transfer A, 42: 465-486.

[10] Oztop, H. F. (2006): Combined convection heat transfer in a porous lid-driven enclosure due to heater with finite length, Int. Commun. Heat Mass Transfer, 33: 772-779.

[11] Rahman, M. M., Alim, M. A., Chowdhury, M. K. (2009): Magnetohydrodynamic Mixed Convection Around a Heat Conducting Horizontal Circular Cylinder in a Rectangular Lid-driven Cavity with Joule Heating, J. Sci. Res., 1(3): 461-472.

[12] Rahman, M. M., Alim, M. A., Sarker, M. M. A. (2010): Numerical study on the conjugate effect of joule heating and magnato-hydrodynamics mixed convection in an obstructed lid-driven square cavity, International Communications in Heat and Mass Transfer, 37: 524-534.

[13] Tanmay Basak, Roy, S., Paul, T., Pop, I.(2006): Natural convection in a square cavity filled with a porous medium: Effects of various thermal boundary conditions, International Journal of Heat and Mass Transfer, 49: 1430-1441. 